\title{
Bound and scattering states with non-local potentials
}

\author{
M. Viviani ${ }^{\mathrm{a}}$, L. Girlanda ${ }^{\mathrm{a}}$, A. Kievsky ${ }^{\mathrm{a}}$, L. E. Marcucci ${ }^{\mathrm{b}}$, R. Schiavilla ${ }^{\mathrm{c}}$, and S. Rosati ${ }^{\mathrm{b}}$

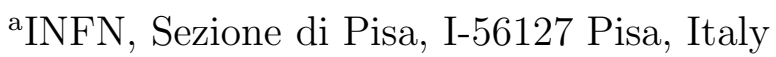 \\ ${ }^{b}$ Department of Physics "E. Fermi", University of Pisa, I-56127 Pisa, Italy \\ cJefferson Lab, Newport News, USA and Phys. Dept., ODU, Norfolk, USA \\ The application of the hyperspherical harmonics method to the case of non-local poten- \\ tials is described. Given the properties of the hyperspherical harmonic functions, there \\ are no difficulties in considering the approach in both coordinate and momentum space. \\ The results for the ${ }^{3} \mathrm{H}$ and ${ }^{4} \mathrm{He}$ binding energies and $n-{ }^{3} \mathrm{H}$ scattering lengths are found \\ to be in good agreement with those obtained with other different techniques. A study of \\ the ${ }^{4} \mathrm{He}$ form factor is also reported, with a careful investigation of the contribution from \\ isospin symmetry violation. Its effect on parity violating elastic scattering of polarized \\ electrons from ${ }^{4} \mathrm{He}$ is investigated. In particular, a simple analysis of the recently mea- \\ sured left-right asymmetry at low $Q^{2}$ shows that the contribution of these isospin admixture \\ are found of comparable magnitude to that associated with strangeness components in the \\ nucleon electric form factor.
}

\section{INTRODUCTION}

A number of "realistic" nucleon-nucleon (NN) potentials have been determined in recent years. They reproduce the deuteron binding energy and fit a large set of NN scattering data below the pion-production threshold with a $\chi^{2} /$ datum $\simeq 1$. Among these potentials we can mention the "phenomenological" models devised in the years 1994-2001 [1-3], the non-local potentials of Doleschall and coll. [4], the potentials based on chiral symmetry [5, $6]$, and a set of "effective" potentials where the repulsive part at short interparticle distance is eliminated [7-9]. These potentials are rather different, as can be seen in Fig. 1, where the momentum-space diagonal (on-shell) part of the ${ }^{3} S_{1}$ interaction is shown.

In general, the "phenomenological" potentials have sizable high momentum tails, due to their configuration-space large repulsion at short interparticle distances. The chiral potentials have a momentum cutoff $\Lambda$ of the order $3-4 \mathrm{fm}^{-1}$, and the "effective" potentials an even lower one $\left(\Lambda \approx 2 \mathrm{fm}^{-1}\right)$. This cutoff is related to the scale at which in each model unknown physics starts to be important. In the "effective" models, the missed physics above the cutoff is taken into account using a renormalization group method and/or some unitary transformation. This procedure changes the properties of the degrees of freedom at the base of each model. Namely, the nucleons interacting via the soft "effective" potentials are particles rather different from the "bare" nucleons entering, for example, in the chiral Lagrangian, which can interact through the exchanges of pions. 


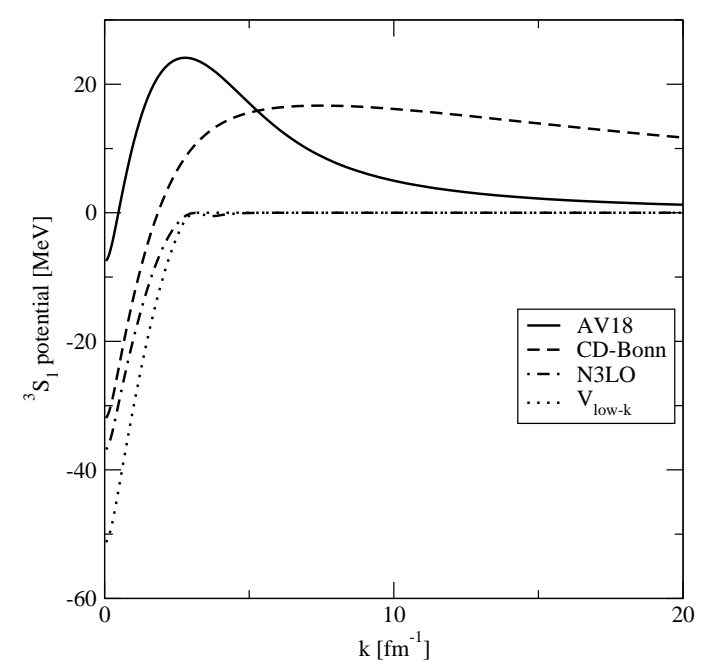

Figure 1. Diagonal part of the ${ }^{3} S_{1}$ potential in momentum space for the AV18 [1], CD Bonn [3], N3LO [5] and one version of the $V_{l o w-k}$ [8] interaction models.

It is therefore of interest to perform tests for understanding which of these different pictures could be more appropriate to describe the very complex nature of the various nuclear systems. A possibility is to study the effects of these potentials in systems with $A \geq 3$ [10]. Clearly in these systems one has to add also proper many-nucleon forces. It is currently believed that only three-nucleon $(3 \mathrm{~N})$ forces should play a relevant role in light systems (for a first quantitative estimate of the magnitude of a four nucleon force, see Ref. [11]). Several models of 3N forces have been proposed, mainly based on the exchange of pions among the three nucleons [12-15]. New mechanisms are also being studied with the chiral approach [16].

Several theoretical methods to solve the Schrödinger equation of $A \geq 3$ systems have been developed during the last decades $[17,18]$. Among these methods, of particular importance are the Faddeev-Yakubovsky (FY) equations approach [19-22], the quantum Monte Carlo methods [15,14], and the techniques based on the expansion of the nuclear wave function on an appropriate basis, like the hyperspherical-harmonics (HH) [23-27], the harmonic oscillator [28], or the gaussian basis [29,30]. Some of these methods are variational while others are not, and they are more or less advantageous depending on the problem at hand. All of them provide very accurate results for $A=3,4$ bound states [31].

In the last few years, considerable effort has been devoted by the authors of the present paper to the development and application of the $\mathrm{HH}$ technique to study bound and scattering states of three or four nucleons with realistic local NN potentials [23,25]. Very recently, we have extended the application of the $\mathrm{HH}$ method also to non-local potentials. The first results obtained for the $A=3$ and 4 bound states have been already illustrated in Ref. [26]. Here, the first (preliminary) results obtained for a scattering problem with a non local potential (the chiral N3LO potential of Ref. [5]) are reported.

The method allows for the determination of very accurate wave functions for the $A=3$ and 4 nuclear systems. As an example of application of such wave functions, we report 
in the last part of the paper a recent calculation of the isospin violation contribution to the parity-violating asymmetry measured in ${ }^{4} \mathrm{He}\left(\vec{e}, e^{\prime}\right){ }^{4} \mathrm{He}$ experiments, a crucial quantity measured for isolating the contribution of the strange electric form factor of the nucleon.

The paper is organized as follows. Some details of the formalism are presented in the next section. In Sec. 3, the results for the ${ }^{4} \mathrm{He}$ binding energies and $n-{ }^{3} \mathrm{H}$ scattering lengths are given and compared with those obtained with other different techniques. In Sec. 4 , the contribution of the isospin-symmetry breaking corrections to the parityviolating asymmetry measured in ${ }^{4} \mathrm{He}\left(\vec{e}, e^{\prime}\right)^{4} \mathrm{He}$ is discussed. A few final remarks and conclusions are given in Sec. 5 .

\section{Formalism}

The state of a $A$-body system can be written as

$\left|\Psi_{A}^{J J_{z} \pi}\right\rangle=\sum_{\mu} c_{\mu}\left|\Phi_{\mu}\right\rangle$

where $\left|\Phi_{\mu}\right\rangle$ are a suitable complete set of states, and $\mu$ is an index denoting the set of quantum numbers necessary to completely determine the basis elements. In the present work, the states $\left|\Phi_{\mu}\right\rangle$ have been written in terms of the $\mathrm{HH}$ functions. In configuration space [26]:

$\left\langle\boldsymbol{x}_{1}, \ldots, \boldsymbol{x}_{N} \mid \Phi_{\mu}\right\rangle=f_{l}(\rho) \mathcal{Y}_{\{G\}}\left(\Omega^{(\rho)}\right)$

where $\boldsymbol{x}_{i}$ are a set of $N=A-1$ Jacobi vectors and $\mathcal{Y}_{\{G\}}(\Omega)$ are a properly antisymmetrized product of $\mathrm{HH}$ functions [32] and spin-isospin states. In the equation above, $\rho$ is the socalled hyperradius, $G$ is the grand angular quantum number, and $\Omega^{(\rho)}$ is a set of angularhyperangular coordinates defined as:

$$
\begin{aligned}
\rho & =\sqrt{\sum_{i=1}^{N} \boldsymbol{x}_{i}^{2}}, & \Omega^{(\rho)}=\left[\hat{\boldsymbol{x}}_{1}, \hat{\boldsymbol{x}}_{2}, \cdots, \hat{\boldsymbol{x}}_{N} ; \phi_{2}, \cdots, \phi_{N}\right], \\
\tan \phi_{i} & =\frac{1}{x_{i}} \sqrt{\sum_{j=1}^{i-1} x_{j}^{2}}, & i=2, \ldots, N .
\end{aligned}
$$

Finally, the suffix $(\rho)$ recalls the use of the coordinate space and the functions $f_{l}(\rho)$ are a suitable set of basis functions [26].

Using the properties of the HH functions [32], it is easy to obtain the momentum space version of the states $\left|\Phi_{\mu}\right\rangle$, namely

$$
\left\langle\boldsymbol{k}_{1}, \ldots, \boldsymbol{k}_{N} \mid \Phi_{\mu}\right\rangle=g_{G, l}(Q) \mathcal{Y}_{\{G\}}\left(\Omega^{(Q)}\right),
$$

where $\mathcal{Y}_{\{G\}}\left(\Omega^{(Q)}\right)$ is the same as $\mathcal{Y}_{\{G\}}\left(\Omega^{(\rho)}\right)$ with $\boldsymbol{x}_{i} \rightarrow \boldsymbol{k}_{i}, \boldsymbol{k}_{i}$ being the Jacobi momenta and $Q=\sqrt{\sum_{i=1}^{N} \boldsymbol{k}_{i}^{2}}$. Finally:

$$
g_{G, l}(Q)=(-i)^{G} \int_{0}^{\infty} d \rho \frac{\rho^{3 N-1}}{(Q \rho)^{3 N / 2-1}} J_{\mathcal{L}+1 / 2}(Q \rho) f_{l}(\rho) \quad \mathcal{L}=G+(3 N-3) / 2 .
$$


It is not difficult to evaluate analytically the above integral for the functions $f_{l}(\rho)$ commonly used [26].

The unknown coefficients $c_{\mu}$ entering the expansion given in Eq. (1) are obtained with the Rayleigh-Ritz variational method for the bound states and the Kohn variational method for the scattering states. To apply these methods, the matrix elements of the interaction with respect to the chosen basis states have to be computed. In our case, it is thus possible to compute in the corresponding space the matrix elements of interaction given in coordinate or momentum space. With two-body local potentials the matrix elements of the interactions can be obtained via two dimensional integrals. The non-locality of the interaction requires a three-dimensional integration, which can be performed using standard numerical methods. Obviously, there are no difficulties in including the local Coulomb interaction. The inclusion of a local $3 \mathrm{~N}$ force requires a three-dimensional integration and we will present here the results obtained with one of the most used models. The inclusion of $3 \mathrm{~N}$ forces derived from chiral perturbation theory is in progress.

\section{Results}

To give an example of the accuracy reached, the converged values for the triton, ${ }^{3} \mathrm{He}$ and ${ }^{4} \mathrm{He}$ binding energies and other ground-state properties obtained with the CD Bonn 2000 [3], N3LO [5], AV18 [1] and AV18 plus Urbana-IX 3N potential [14] (AV18/UIX) interaction models are listed in Table 1, 2 and 3, respectively. The numerical uncertainty in the triton and ${ }^{3} \mathrm{He}$ binding energy has been estimated to be at the most of the order of $1 \mathrm{keV}$, and in the ${ }^{4} \mathrm{He}$ binding energy of the order of $10 \mathrm{keV}$. These results are compared with those obtained with other approaches. In particular, we have considered the Faddeev (for $A=3$ ) and FY (for $A=4$ ) approach [33-37], the Effective Interaction Hyperspherical Harmonic (EIHH) method [38], the GFMC approach [39], and the No Core Shell Model (NCSM) method $[28,40]$. In the ${ }^{3} \mathrm{He}$ and ${ }^{4} \mathrm{He}$ case, all the results for the CD Bonn 2000 and N3LO potentials have been obtained including the point Coulomb interaction, except for the ${ }^{4} \mathrm{He}$ FY results with the N3LO potential. The latter have been obtained including a more complicated electro-magnetic interaction between nucleons [34], whose effects are however quite similar to those of the point Coulomb interaction.

There is a good agreement, at the level of $0.1 \%$, among the results obtained with different techniques for all quantities considered in the tables. Furthermore, the mean value of the kinetic energy for the N3LO case is smaller than that found with the AV18 potential. This is due to the fact that the repulsion at short interparticle distances is softer for the N3LO potential than for the AV18 potential. Also the percentages of the $P$ and $D$-waves is significantly smaller for the N3LO potential. We have observed a faster convergence of the $\mathrm{HH}$ expansion in this case with respect to the other potentials.

We now consider the results for the $T=3 / 2$ and $T>0$ components in the $A=3$ and 4 systems, respectively. The percentage of the $T=1$ component in the ${ }^{4} \mathrm{He}$ ground state wave function is almost independent from the adopted potential model. In fact, as discussed in Ref. [25], 50\% of it is due to the effect of the Coulomb interaction between the protons, the remaining $50 \%$ is due to the isospin symmetry breaking (ISB) terms in the nuclear interaction. Consequently, the difference in $P_{T=1}$ for the various two-body potentials is small. On the contrary, the $T=3 / 2$ component in triton and the $T=2$ 
Table 1

The triton binding energies $B(\mathrm{MeV})$, the mean square radii $\sqrt{\left\langle r^{2}\right\rangle}(\mathrm{fm})$, the expectation values of the kinetic energy operator $\langle T\rangle(\mathrm{MeV})$, and the mixed-symmetry $S^{\prime}, P, D$, and isospin $T=3 / 2$ probabilities (all in \%), calculated with the CD Bonn 2000, N3LO, AV18 and AV18/UIX interaction models, are compared with the results obtained within the Faddeev equations approach [33-36] (FE) and within the No Core Shell Model (NCSM) approach $[28,40]$. The results obtained in Ref. [33] within the correlated-hypersphericalharmonics $(\mathrm{CHH})$ approach [24] have been reported for sake of comparison.

\begin{tabular}{llccccccc}
\hline Interaction & Method & $B$ & $\langle T\rangle$ & $\sqrt{\left\langle r^{2}\right\rangle}$ & $P_{S^{\prime}}$ & $P_{P}$ & $P_{D}$ & $P_{T=3 / 2}$ \\
\hline CD Bonn 2000 HH & 7.998 & 37.630 & 1.721 & 1.31 & 0.047 & 7.02 & 0.0049 \\
& FE [33,34] & 7.997 & 37.620 & - & 1.31 & 0.047 & 7.02 & 0.0048 \\
& FE [35,36] & 7.998 & 37.627 & - & 1.31 & 0.047 & 7.02 & 0.0048 \\
& NCSM [28,40] & $7.99(1)$ & - & - & - & - & - & - \\
\hline N3LO & HH & 7.854 & 34.555 & 1.758 & 1.36 & 0.037 & 6.31 & 0.0009 \\
& FE [33,34] & 7.854 & 34.546 & - & 1.37 & 0.037 & 6.32 & 0.0009 \\
& FE [35,36] & 7.854 & 34.547 & - & 1.37 & 0.037 & 6.32 & 0.0009 \\
& NCSM [28,40] & $7.85(1)$ & - & - & - & - & - & - \\
\hline AV18 & HH & 7.623 & 46.729 & 1.770 & 1.294 & 0.066 & 8.509 & 0.0025 \\
& CHH [33] & 7.624 & 46.727 & - & 1.293 & 0.066 & 8.510 & 0.0025 \\
& FE [33] & 7.621 & 46.73 & - & 1.291 & 0.066 & 8.510 & 0.0025 \\
\hline AV18/UIX & HH & 8.479 & 51.275 & 1.684 & 1.054 & 0.135 & 9.301 & 0.0025 \\
& FE [33] & 8.476 & 51.28 & - & 1.052 & 0.135 & 9.302 & 0.0025 \\
\hline
\end{tabular}

component in ${ }^{4} \mathrm{He}$ are largely dominated by ISB of nuclear origin (different pion masses, etc.). The values reported in Table 1 and 3 show that, depending on the interaction, rather different values for the triton $P_{T=3 / 2}$ and the ${ }^{4} \mathrm{He} P_{T=2}$ are obtained (note that the standard models of TNI have little effect on the isospin admixtures [25]). The ISB magnitude in the two-body interaction is fixed by fitting the $N N$ scattering data, and therefore comes from differences observed in the $p p$ and $n p$ systems. In particular, the CD Bonn 2000 and N3LO potentials fit the same $N N$ data set [41]. The origin of the rather large differences found for the triton $P_{T=3 / 2}$ and the ${ }^{4} \mathrm{He} P_{T=2}$ (a factor 5 between CD Bonn 2000 and N3LO) must be related to quite different off-shell behavior of the ISB terms of both interactions. Note that the $T=3 / 2$ component in ${ }^{3} \mathrm{He}$ is also affected by the Coulomb interaction, which reduces by more than a factor of 2 the difference between the CD Bonn 2000 and N3LO $P_{T=3 / 2}$ results.

Finally, we observe that knowledge of the $T=1$ percentage is important for parityviolating electron scattering experiments on ${ }^{4} \mathrm{He}$, aimed at studying admixture of strange quark $s \bar{s}$ pairs. This topic will be discussed in the next section.

Next, we report some (preliminary) scattering lengths obtained with the N3LO potential. In Fig. 2, we report the results for the $n-d$ doublet scattering length, plotted versus the corresponding ${ }^{3} \mathrm{H}$ binding energy. The experimental value is from Ref. [42]. It has been observed a long time ago that the doublet scattering length is correlated to the ${ }^{3} \mathrm{H}$ binding energy. This correlation is known as the Phillips line [43]. It was found that this 
Table 2

Same as Table 1 but for ${ }^{3} \mathrm{He}$.

\begin{tabular}{llccccccc}
\hline Interaction & Method & $B$ & $\langle T\rangle$ & $\sqrt{\left\langle r^{2}\right\rangle}$ & $P_{S^{\prime}}$ & $P_{P}$ & $P_{D}$ & $P_{T=3 / 2}$ \\
\hline CD Bonn 2000 HH & 7.262 & 36.777 & 1.759 & 1.54 & 0.046 & 7.00 & 0.0109 \\
& FE [33,34] & 7.261 & 36.756 & - & 1.54 & 0.046 & 7.00 & 0.0110 \\
& FE [35,36] & 7.263 & 36.761 & - & 1.54 & 0.046 & 7.00 & 0.0110 \\
\hline N3LO & HH & 7.128 & 33.789 & 1.797 & 1.61 & 0.037 & 6.31 & 0.0062 \\
& FE [33,34] & 7.128 & 33.775 & - & 1.61 & 0.037 & 6.31 & 0.0063 \\
& FE [35,36] & 7.128 & 33.775 & - & 1.61 & 0.037 & 6.32 & 0.0063 \\
\hline AV18 & HH & 6.924 & 45.690 & 1.808 & 1.530 & 0.065 & 8.466 & 0.0080 \\
& CHH [33] & 6.925 & 45.685 & - & 1.530 & 0.065 & 8.467 & 0.0080 \\
& FE [33] & 6.923 & 45.68 & - & 1.524 & 0.065 & 8.466 & 0.0081 \\
\hline AV18/UIX & HH & 7.750 & 50.211 & 1.716 & 1.242 & 0.132 & 9.248 & 0.0075 \\
& FE [33] & 7.746 & 50.21 & - & 1.235 & 0.132 & 9.248 & 0.0075 \\
\hline
\end{tabular}

Table 3

The $\alpha$-particle binding energies $B(\mathrm{MeV})$, the expectation values of the kinetic energy operator $\langle T\rangle(\mathrm{MeV})$, the mean square radii $\sqrt{\left\langle r^{2}\right\rangle}(\mathrm{fm})$, and the $P, D, T=1$ and $T=2$ probabilities (\%) for four NN potential models.

\begin{tabular}{llccccccc}
\hline Interaction & Method & $B$ & $\langle T\rangle$ & $\sqrt{\left\langle r^{2}\right\rangle}$ & $P_{P}$ & $P_{D}$ & $P_{T=1}$ & $P_{T=2}$ \\
\hline CD Bonn & HH & 26.13 & 77.58 & 1.454 & 0.223 & 10.74 & 0.0029 & 0.0108 \\
& FY [34] & 26.16 & 77.59 & - & 0.225 & 10.77 & 0.0030 & 0.0108 \\
\hline N3LO & HH & 25.38 & 69.24 & 1.516 & 0.172 & 9.289 & 0.0035 & 0.0024 \\
& FY [34] & 25.37 & 69.20 & - & 0.172 & 9.293 & 0.0033 & 0.0024 \\
& NCSM [28] & $25.36(4)$ & - & - & - & - & - & - \\
\hline AV18 & HH & 24.210 & 97.84 & 1.512 & 0.347 & 13.74 & 0.0028 & 0.0052 \\
& FY [20] & 24.25 & 97.80 & - & 0.35 & 13.78 & 0.003 & 0.005 \\
& FY [37] & 24.223 & 97.77 & 1.516 & - & - & - & - \\
& EIHH [38] & 24.268 & - & 1.518 & - & - & - & - \\
\hline AV18/UIX & HH & 28.47 & 113.30 & 1.428 & 0.73 & 16.03 & 0.0025 & 0.0050 \\
& FY [20] & 28.50 & 113.21 & - & 0.75 & 16.03 & - & - \\
& GFMC [39] & $28.34(4)$ & $110.7(7)$ & 1.44 & - & - & - & - \\
\hline
\end{tabular}




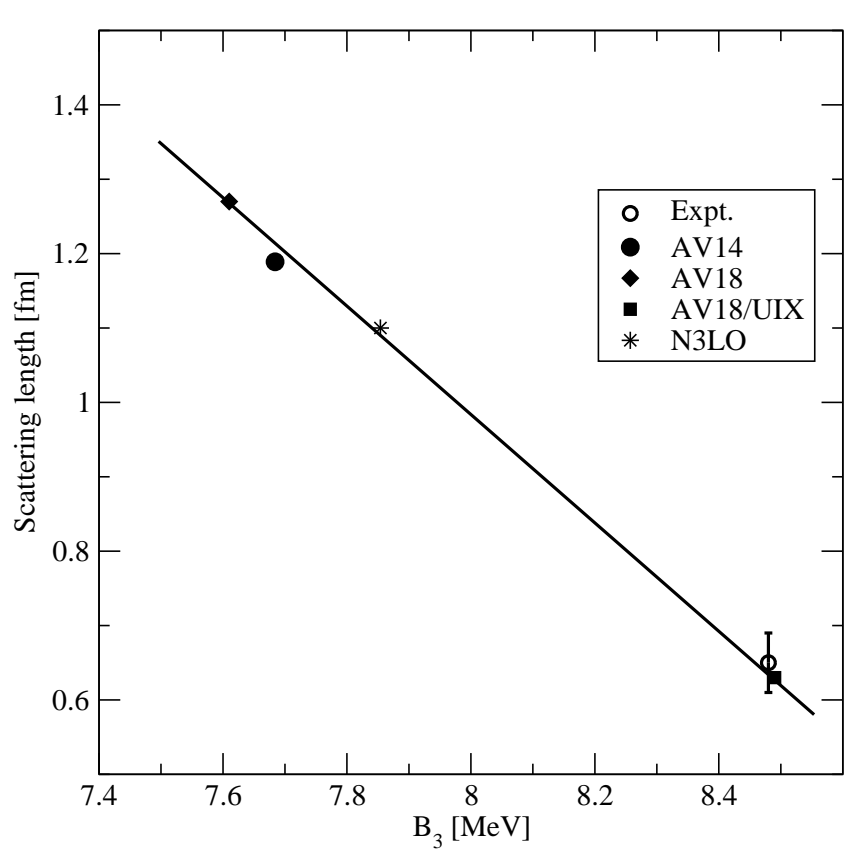

Figure 2. $n-d$ doubled scattering length plotted against the ${ }^{3} \mathrm{H}$ binding energy. The open circle is the experimental value of Ref. [42]. The straight line is a linear fit of the theoretical results.

line passes through the experimental point [44]. We have found that also the N3LO result lies on the same line (see Fig. 2)

Analogously, the $n-{ }^{3} \mathrm{H}$ singlet $a_{s}$ and triplet $a_{t}$ scattering lengths with the N3LO and other potential models are reported in Fig. 3. The most recent experimental values $[45,46]$ of $a_{s}$ and $a_{t}$ have also been reported. We have used the AV14 [47], AV8 [48] and AV18 [1] potentials. Including 3N forces we have: the AV18+Urbana IX (AV18/UIX) [14], AV14+Brazil with $\Lambda=5.6 m_{\pi}$ (AV14BR1) and AV14+Brazil with $\Lambda=5.8 m_{\pi}$ (AV14BR2) [13]. In the AV18UR model, one the parameters of the $3 \mathrm{~N}$ potential was chosen to reproduce the experimental ${ }^{3} \mathrm{H}$ binding energy value $B_{3}=8.48 \mathrm{MeV}$. The AV14BR1 and AV14BR2 models have been chosen so as to give slightly larger $B_{3}$ values. It was already observed in Ref. [49] that the calculated singlet and triplet scattering lengths fall essentially on straight lines. Also in this case, we have found that also the N3LO results lie on the same lines (see Fig. 3). However, the experimental values extracted from the data do not fall on the theoretical curves. This disagreement is related to a rather small discrepancy between the calculated and measured coherent scattering length. The origin of this discrepancy is still not clear.

\section{Isospin mixing in the nucleon and ${ }^{4} \mathrm{He}$ and the nucleon strange electric form factor}

One of the challenges of modern hadronic physics is to determine, at a quantitative level, the role that quark-antiquark pairs, and in particular $s \bar{s}$ pairs, play in the structure 


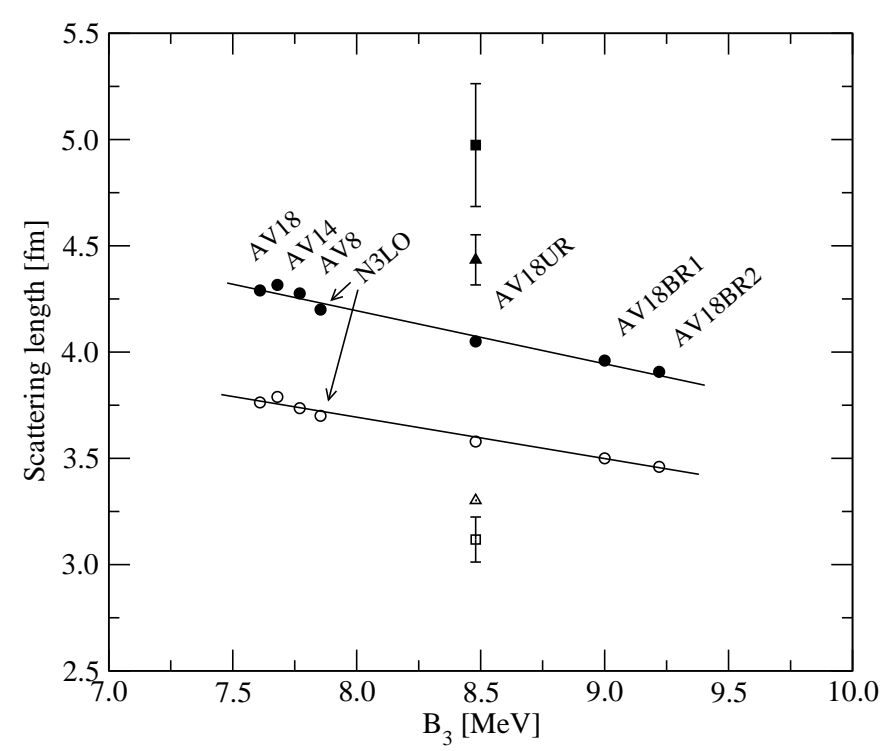

Figure 3. Singlet (full symbols) and triplet (open symbols) $n-{ }^{3} \mathrm{H}$ scattering lengths plotted against the ${ }^{3} \mathrm{H}$ binding energy. The squares (triangles) are the experimental values of ref. [45] (ref. [46]). The straight lines are linear fits of the theoretical results.

of the nucleon. Parity-violating (PV) electron scattering from nucleons and nuclei offers the opportunity to investigate this issue experimentally. The PV asymmetry $\left(A_{P V}\right)$ arises from interference between the amplitudes due to exchange of photons and $Z$-bosons, which couple respectively to the electromagnetic (EM) and weak neutral (NC) currents. These currents involve different combinations of quark flavors, and therefore measurements of $A_{P V}$, in combination with electromagnetic form factor data for the nucleon, allow one to isolate the electric and magnetic form factors $G_{E}^{s}$ and $G_{M}^{s}$, associated with the strangequark content of the nucleon.

Experimental determinations of these form factors have been reported recently by the Jefferson Lab HAPPEX [50] and G0 [51] Collaborations, Mainz A4 Collaboration [52], and MIT-Bates SAMPLE Collaboration [53]. These experiments have scattered polarized electrons from either unpolarized protons at forward angles [50-52] or unpolarized protons and deuterons at backward angles [53]. The resulting PV asymmetries are sensitive to different linear combinations of $G_{E}^{s}$ and $G_{M}^{s}$ as well as the nucleon axial-vector form factor $G_{A}^{Z}$. However, no robust evidence has emerged so far for the presence of strange-quark effects in the nucleon.

Last year, the HAPPEX Collaboration $[54,55]$ at Jefferson Lab reported on measurements of the PV asymmetry in elastic electron scattering from ${ }^{4} \mathrm{He}$ at four-momentum transfers of $0.091(\mathrm{GeV} / \mathrm{c})^{2}$ and $0.077(\mathrm{GeV} / \mathrm{c})^{2}$. Because of the $J^{\pi}=0^{+}$spin-parity assignments of this nucleus, transitions induced by magnetic and axial-vector currents are forbidden, and therefore these measurements can lead to a direct determination of the strangeness electric form factor $G_{E}^{s}[56,57]$, provided that isospin symmetry breaking effects in both the nucleon and ${ }^{4} \mathrm{He}$, and relativistic and meson-exchange contributions to 
the nuclear EM and weak vector charge operators, are negligible. A realistic calculation of these latter contributions [57] found that they are in fact tiny at low momentum transfers. The goal of the present letter is to provide a quantitative estimate of the ISB corrections to the PV asymmetry.

In the following analysis, we only need to consider the time components of the EM current and vector part of the weak NC current - the weak vector charge referred to above [57]. We account for isospin symmetry breaking in both the nucleon and $\alpha$-particle. We first discuss it in the nucleon. Ignoring radiative corrections, the EM and weak vector charge operators can be decomposed as

$j_{\mathrm{EM}}^{\mu=0}=j^{(0)}+j^{(1)}$,

$j_{\mathrm{NC}}^{\mu=0}=-4 \sin ^{2} \theta_{W} j^{(0)}+\left(2-4 \sin ^{2} \theta_{W}\right) j^{(1)}-j^{(s)}$,

where $j^{(0)}$ and $j^{(1)}$ are respectively the isoscalar and isovector components of the EM charge operators, $j^{(s)}$ is the (isoscalar) component due to strange-quark contributions, and $\sin ^{2} \theta_{W}$ contains the Weinberg mixing angle. In a notation similar to that adopted by the authors of Ref. [58], we introduce form factors corresponding to the following matrix elements of $j^{(0)}$ and $j^{(1)}$ between proton $(p)$ and neutron $(n)$ states:

$$
\begin{aligned}
\left\langle p\left|j^{(0)}\right| p\right\rangle & \rightarrow G_{E}^{0}\left(Q^{2}\right)+G_{E}^{\emptyset}\left(Q^{2}\right), \\
\left\langle n\left|j^{(0)}\right| n\right\rangle & \rightarrow G_{E}^{0}\left(Q^{2}\right)-G_{E}^{\emptyset}\left(Q^{2}\right), \\
\left\langle p\left|j^{(1)}\right| p\right\rangle & \rightarrow G_{E}^{1}\left(Q^{2}\right)+G_{E}^{x}\left(Q^{2}\right), \\
\left\langle n\left|j^{(1)}\right| n\right\rangle & \rightarrow-G_{E}^{1}\left(Q^{2}\right)+G_{E}^{\chi}\left(Q^{2}\right),
\end{aligned}
$$

where the arrow indicates that only leading contributions are listed in the non-relativistic limit of these matrix elements. While higher order corrections associated with the DarwinFoldy and spin-orbit terms are not displayed explicitly in the equations above, they are in fact retained in the calculations discussed later in the present work. The form factors $G_{E}^{\emptyset}\left(Q^{2}\right)$ and $G_{E}^{\chi}\left(Q^{2}\right)$ parameterize ISB effects in the nucleon states. We also introduce the strange form factor via

$$
\left\langle p\left|j^{(s)}\right| p\right\rangle=\left\langle n\left|j^{(s)}\right| n\right\rangle \rightarrow G_{E}^{s}\left(Q^{2}\right),
$$

where here ISB terms in the $p, n$ states are neglected. Contributions from sea quarks heavier than strange are also ignored.

In terms of the experimental proton and neutron electric form factors, derived from the matrix elements $\left\langle p\left|j_{\mathrm{EM}}^{\mu=0}\right| p\right\rangle \rightarrow G_{E}^{p}\left(Q^{2}\right)$ and $\left\langle n\left|j_{\mathrm{EM}}^{\mu=0}\right| n\right\rangle \rightarrow G_{E}^{n}\left(Q^{2}\right)$, we obtain:

$$
\begin{aligned}
& G_{E}^{0}=\left(G_{E}^{p}+G_{E}^{n}\right) / 2-G_{E}^{\chi}, \\
& G_{E}^{1}=\left(G_{E}^{p}-G_{E}^{n}\right) / 2-G_{E}^{\emptyset},
\end{aligned}
$$

where the $Q^{2}$ dependence in these and the following two equations is understood. In the limit in which the $p, n$ states form an isospin doublet, the form factors $G_{E}^{\emptyset}$ and $G_{E}^{\wedge}$ vanish, and $G_{E}^{0}$ and $G_{E}^{1}$ reduce to the standard isoscalar and isovector combinations of the proton 
and neutron electric form factors. The proton and neutron vector NC form factors follow from Eq. (8), i.e.

$$
\begin{aligned}
& G_{E}^{p, Z}=-4 \sin ^{2} \theta_{W} G_{E}^{p}+\left(G_{E}^{p}-G_{E}^{n}\right)+2\left(G_{E}^{\chi}-G_{E}^{\emptyset}\right)-G_{E}^{s}, \\
& G_{E}^{n, Z}=-4 \sin ^{2} \theta_{W} G_{E}^{n}-\left(G_{E}^{p}-G_{E}^{n}\right)+2\left(G_{E}^{\chi}+G_{E}^{\emptyset}\right)-G_{E}^{s} .
\end{aligned}
$$

We now turn to the nuclear charge operator. At low momentum transfer, it is simply given by

$\rho^{(\mathrm{EM})}(\mathbf{q})=G_{E}^{p}\left(Q^{2}\right) \sum_{k=1}^{Z} e^{i \mathbf{q} \cdot \mathbf{r}_{k}}+G_{E}^{n}\left(Q^{2}\right) \sum_{k=Z+1}^{A} e^{i \mathbf{q} \cdot \mathbf{r}_{k}}$,

where $Z$ is the number of protons, $A-Z$ the number of neutrons, and for elastic scattering from a nuclear target of mass $m_{A}$ the squared four-momentum transfer is taken as $Q^{2}=$ $2 m_{A}\left(\sqrt{q^{2}+m_{A}^{2}}-m_{A}\right)$, with $\mathbf{q}$ being the three-momentum transfer, and $q=|\mathbf{q}|$. An equation similar to Eq. (18) holds for the weak vector charge operator, but with $G_{E}^{p}$ and $G_{E}^{n}$ being replaced respectively by $G_{E}^{p, Z}$ and $G_{E}^{n, Z}$. It is also convenient to define the charge operators:

$$
\begin{aligned}
& \rho^{(0)}(\mathbf{q})=\frac{G_{E}^{p}+G_{E}^{n}}{2} \sum_{k=1}^{A} e^{i \mathbf{q} \cdot \mathbf{r}_{k}}, \\
& \rho^{(1)}(\mathbf{q})=\frac{G_{E}^{p}-G_{E}^{n}}{2}\left(\sum_{k=1}^{Z} e^{i \mathbf{q} \cdot \mathbf{r}_{k}}-\sum_{k=Z+1}^{A} e^{i \mathbf{q} \cdot \mathbf{r}_{k}}\right),
\end{aligned}
$$

from which

$$
\begin{aligned}
\rho^{(\mathrm{EM})}(\mathbf{q})= & \rho^{(0)}(\mathbf{q})+ \\
\rho^{(\mathrm{NC})}(\mathbf{q})=-4 \sin ^{2} \theta_{W} \rho^{(\mathrm{EM})}(\mathbf{q}) & +\frac{2 G_{E}^{x}-G_{E}^{s}}{\left(G_{E}^{p}+G_{E}^{n}\right) / 2} \rho^{(0)}(\mathbf{q}) \\
& +2 \rho^{(1)}(\mathbf{q})-\frac{2 G^{\emptyset}}{\left(G_{E}^{p}-G_{E}^{n}\right) / 2} \rho^{(1)}(\mathbf{q}),
\end{aligned}
$$

where again the $Q^{2}$ dependence of the nucleon form factors has been suppressed here and in the following for brevity. The relations above lead to the definition of the following nuclear form factors:

$$
\left\langle{ }^{4} \mathrm{He}\left|\rho^{(a)}(\mathbf{q})\right|{ }^{4} \mathrm{He}\right\rangle \equiv \sqrt{4 \pi} F^{(a)}(q), \quad a=\mathrm{EM}, 0,1,
$$

having the normalizations $F^{(\mathrm{EM})}(0)=F^{(0)}(0)=Z / \sqrt{4 \pi}$ and $F^{(1)}(0)=0$. The form factor $F^{(1)}(q)$ is very small because ${ }^{4} \mathrm{He}$ is predominantly an isoscalar state. Thus, ignoring second order terms like $G^{\emptyset} F^{(1)}(q)$, we obtain for the PV asymmetry measured in $\left(\vec{e}, e^{\prime}\right)$ elastic scattering from ${ }^{4} \mathrm{He}$ :

$$
A_{P V}=\frac{G_{\mu} Q^{2}}{4 \pi \alpha \sqrt{2}}\left[4 \sin ^{2} \theta_{W}-2 \frac{F^{(1)}(q)}{F^{(0)}(q)}-\frac{2 G_{E}^{\chi}-G_{E}^{s}}{\left(G_{E}^{p}+G_{E}^{n}\right) / 2}\right],
$$


where $G_{\mu}$ is the Fermi constant as determined from muon decays, and $\sin ^{2} \theta_{W}$ incorporates radiative corrections. The terms $G_{E}^{\chi}$ and $F^{(1)}(q) / F^{(0)}(q)$ are the contributions to $A_{P V}$, associated with the violation of isospin symmetry at the nucleon and nuclear level, respectively.

The most accurate measurement of $A_{P V}$ has been recently reported at $Q^{2}=0.077(\mathrm{GeV} / \mathrm{c})^{2}[55]$ :

$A_{P V}=[+6.40 \pm 0.23$ (stat) \pm 0.12 (syst) $] \mathrm{ppm}$,

from which, after inserting the values for $G_{\mu}=1.16637 \times 10^{-5} \mathrm{GeV}^{-2}, \alpha=1 / 137.036$, and $\sin ^{2} \theta_{W}=0.2286$ with its radiative corrections [56] in Eq. (24), one obtains

$\Gamma \equiv-2 \frac{F^{(1)}(q)}{F^{(0)}(q)}-\frac{2 G_{E}^{\chi}-G_{E}^{s}}{\left(G_{E}^{p}+G_{E}^{n}\right) / 2}=0.010 \pm 0.038$

at $Q^{2}=0.077(\mathrm{GeV} / \mathrm{c})^{2}$. This result is consistent with zero. In the following, we discuss the estimates for the ISB corrections first in the nucleon and then in ${ }^{4} \mathrm{He}$, respectively $G_{E}^{x}\left(Q^{2}\right)$ and $F^{(1)}(q)$, at $Q^{2}=0.077(\mathrm{GeV} / \mathrm{c})^{2}$ (corresponding to $q=1.4 \mathrm{fm}^{-1}$ ).

For $G_{E}^{1}\left(Q^{2}\right)$ we use the estimate obtained in Ref. [58], combining a leading-order calculation in chiral perturbation theory with estimates for low-energy constants using resonance saturation. At the specific kinematical point of interest $Q^{2}=0.077(\mathrm{GeV} / \mathrm{c})^{2}$, it results that $G_{E}^{x}\left(Q^{2}\right)=-0.0017 \pm 0.0006$, and with $G_{E}^{p}\left(Q^{2}\right)=0.799$ and $G_{E}^{n}\left(Q^{2}\right)=0.027$ [59], we obtain

$-\frac{2 G_{E}^{1}}{\left(G_{E}^{p}+G_{E}^{n}\right) / 2}=0.008 \pm 0.003$

at $Q^{2}=0.077(\mathrm{GeV} / \mathrm{c})^{2}$.

We now turn to the nuclear ISB corrections. An approximate calculation of the ratio $F^{(1)}(q) / F^{(0)}(q)$ was carried out more than a decade ago [60], by i) taking into account only the isospin admixtures induced by the Coulomb interaction, ii) constructing a $T=1$ $J^{\pi}=0^{+}$breathing mode excitation based on a plausible ansatz, and iii) generating the relevant $T=1$ component in the ${ }^{4} \mathrm{He}$ ground state in first order perturbation theory. The calculated value was found to be rather small, and it produced a less than $1 \%$ correction with respect to the $4 \sin ^{2} \theta_{W}$ term in Eq. (24) at low $Q^{2}$.

Since that pioneering study, significant progress has occurred on several fronts. First, there now exist a number of accurate models of NN potentials $[2,1,3,5,6]$ which include explicit ISB induced by both the strong and electromagnetic interactions. These ISB terms have been constrained by fitting $p p$ and $n p$ elastic scattering data. Second, several accurate methods have been developed to compute ${ }^{4} \mathrm{He}$ wave functions starting from a given realistic nuclear Hamiltonian [31]. In this paper, we use the HH expansion method to compute the ${ }^{4} \mathrm{He}$ wave function as discussed in the previous sections.

In order to have an estimate of the model dependence, we consider a variety of Hamiltonian models, including: i) the AV18 NN potential [1]; ii) the AV18 plus Urbana-IX 3N potential [14] (AV18/UIX); iii) the CD Bonn [3] NN plus Urbana-IXb 3N potentials (CDBonn/UIXb); and iv) the chiral N3LO [5] NN potential (N3LO). The Urbana UIXb 3N potential is a slightly modified version of the Urbana UIX (in the UIXb, the parameter $U_{0}$ of the central repulsive term has been reduced by the factor 0.812 ), designed to reproduce, 
when used in combination with the CD Bonn potential, the experimental binding energy of ${ }^{3} \mathrm{H}$.

The form factors $F^{(0)}(q)$ and $F^{(1)}(q)$, defined in Eq. (23) and calculated with the AV18/UIX Hamiltonian model, are displayed in Fig. 4, where the effect of inclusion of meson-exchange contributions (MEC) is also shown. The dashed curves are the calculations including only the one-body operators given in Eqs. (19) and (20). The solid curves have been obtained including the relativistic one-body and MEC [57] in the electromagnetic charge operators of Eqs. (19) and (20) (note that ISB corrections in the nucleon form factors entering these contributions are neglected). The experimental data are from Refs. [61]. From the figure it is evident that for $q \leq 1.5 \mathrm{fm}^{-1}$, the effect of MEC in both $F^{(0)}(q)$ and $F^{(1)}(q)$ is negligible.

In the inset of Fig. 4, we also show the the model dependence of the ratio $\left|F^{(1)}(q) / F^{(0)}(q)\right|$ (all calculations include MEC). The various Hamiltonian models give predictions quite close to each other; the remaining differences reflect the different percentages of the $T=1$ component in the ${ }^{4} \mathrm{He}$ wave function.

The calculated ratios $F^{(1)}(q) / F^{(0)}(q)$ at $Q^{2}=0.077(\mathrm{GeV} / \mathrm{c})^{2}$ are of the order of -0.002 . Note that the value estimated previously in Ref. [60] was $\approx-0.001$ at $Q^{2}=0.077(\mathrm{GeV} / \mathrm{c})^{2}$, not far from our result. The value corresponding to the N3LO is somewhat larger than for the other models, as can be seen in Fig. 4, reflecting the larger percentage of $T=1$ admixtures predicted by the N3LO potential. The inclusion of $3 \mathrm{~N}$ potentials tends to decrease the magnitude of $F^{(1)} / F^{(0)}$, and relativistic and MEC are, at this value of $Q^{2}$, negligible.

Therefore, at $Q^{2}=0.077(\mathrm{GeV} / \mathrm{c})^{2}$, both contributions $F^{(1)} / F^{(0)}$ and $G_{E}^{x}$ are found of the same order of magnitude as the central value of $\Gamma$ in Eq. (26). Using in this equation the value $F^{(1)} / F^{(0)} \approx-0.00157$ obtained with the Hamiltonian models including $3 \mathrm{~N}$ potentials, and the chiral result for $G_{E}^{x}=-0.0017 \pm 0.0006$, one would obtain $G_{E}^{s}\left[Q^{2}=\right.$ $\left.0.077(\mathrm{GeV} / \mathrm{c})^{2}\right]=-0.001 \pm 0.016$ thus suggesting that the value of $\Gamma$ is almost entirely due to isospin admixtures. Of course, the experimental error on $\Gamma$ is still too large to allow us to draw a more definite conclusion. A recent estimate of $G_{E}^{s}$ using lattice QCD input obtains [62] $G_{E}^{s}\left(0.1(\mathrm{GeV} / \mathrm{c})^{2}\right)=+0.001 \pm 0.004 \pm 0.003$. An increase of the experimental accuracy of one order of magnitude would be necessary in order to be sensitive to $G_{E}^{s}$ at low values of $Q^{2}$. Indeed, if the lattice QCD prediction above is confirmed, the present data would suggest that the leading correction to the PV asymmetry is from isospin admixtures in the nucleon and/or ${ }^{4} \mathrm{He}$.

\section{Conclusions and perspectives}

The $A=4$ bound state and $n-{ }^{3} \mathrm{H}$ zero energy scattering have been studied with local and non-local two-body potentials using the $\mathrm{HH}$ method. The variational wave function is written as an expansion over a complete basis, which is constructed in coordinate space as a product of hyperradial, $\mathrm{HH}$ and spin-isospin functions. Given the properties of the $\mathrm{HH}$ functions, its Fourier transform can be easily calculated. Thus, the method can be applied in momentum space, as well. For bound states we have calculated the ${ }^{4} \mathrm{He}$ binding energy for the AV18, CD Bonn and N3LO NN potential models. In all cases we have found very good agreement with the results obtained by other groups. We have also given preliminary 


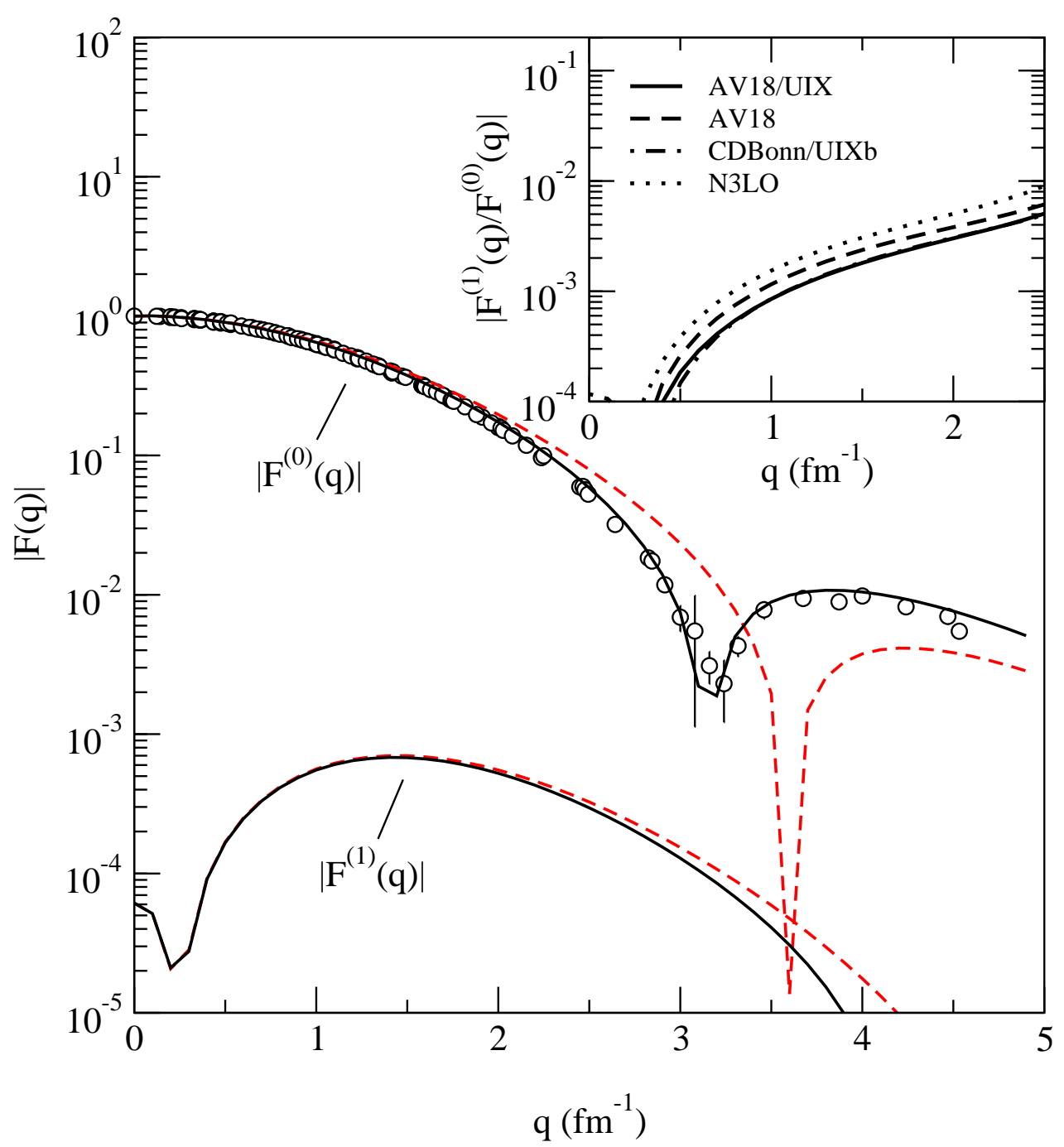

Figure 4. The $F^{(0)}(q)$ and $F^{(1)}(q)$ form factors for the AV18/UIX Hamiltonian model. The $F^{(0)}(q)$ is compared with the experimental ${ }^{4} \mathrm{He}$ charge form factor [61]. See the text for explanation. The ratio $\left|F^{(1)}(q) / F^{(0)}(q)\right|$ (all calculations include MEC) is shown in the inset for the four Hamiltonian models considered in this paper. 
estimates of the $n-d$ and $n-{ }^{3} \mathrm{H}$ scattering lengths with the N3LO potential. These estimates have been found to lie on the same straight lines when plotted against the ${ }^{3} \mathrm{H}$ binding energy as already observed for several other local potential models.

We have also presented estimates for the ISB corrections to the parity-violating asymmetry in electron scattering experiments on ${ }^{4} \mathrm{He}$. The present study shows that such corrections are larger than previously estimated. Indeed, if the present lattice QCD predictions for the strange electric form factor are confirmed, the present data would suggest that the leading correction to the asymmetry comes from isospin admixtures in the nucleon and ${ }^{4} \mathrm{He}$. Also at the nuclear level, the ISB contributions can be related to properties of the $u$ and $d$ quarks, namely their mass and charge differences [63]. Therefore, the experimental study of the parity-violating asymmetry in electron scattering experiments on ${ }^{4} \mathrm{He}$ could give some information on these fundamental properties of the quarks.

In conclusion, the preliminary applications of the $\mathrm{HH}$ method to treat non-local bound and scattering states have been found to be rather successful. We have presented here some results for bound states and the $A=3$ and 4 scattering lengths. The method is now being applied to higher energies.

\section{REFERENCES}

1. R.B. Wiringa, V.G.J. Stoks, and R. Schiavilla, Phys. Rev. C 51, 38 (1995).

2. V.G.J. Stoks et al., Phys. Rev. C 49, 2950 (1994).

3. R. Machleidt, Phys. Rev. C 63, 024001 (2001).

4. P. Doleschall et al., Phys. Rev. C 67, 064005 (2003).

5. D.R. Entem and R. Machleidt, Phys. Rev. C 68, 041001 (2003).

6. E. Epelbaum, W. Gloeckle, and Ulf-G. Meissner, Nucl. Phys. A747, 362 (2005).

7. S.K. Bogner, T.T.S. Kuo, and A. Schwenk, Phys. Rep. 386, 1 (2003).

8. L. Coraggio et al., Phys. Rev. C 71, 014307 (2005).

9. A.M. Shirokov et al., Phys. Rev. C 70, 044005 (2004).

10. N. Barnea, W. Leidemann, and G. Orlandini, Phys. Rev. C 74, 034003 (2006).

11. D. Rozpedzik et al., nucl-th/0606017.

12. S. A. Coon et al., Nucl. Phys. A317, 242 (1979).

13. M. R. Robilotta and H. T. Coelho, Nucl. Phys. A460, 645 (1986).

14. B. S. Pudliner et al., Phys. Rev. C 56, 1720 (1997).

15. S. C. Pieper et al., Phys. Rev. C 64, 014001 (2001).

16. E. Epelbaum et al., Phys. Rev. C 66, 064001 (2002).

17. W. Glöckle et al., Phys. Rep. 274, 107 (1996).

18. J. Carlson and R. Schiavilla, Rev. Mod. Phys. 70, 743 (1998).

19. A. Nogga, H. Kamada, and W. Glöckle, Phys. Rev. Lett. 85, 944 (2000).

20. A. Nogga et al., Phys. Rev. C 65, 054003 (2002).

21. F. Ciesielski, J. Carbonell, and C. Gignoux, Phys. Lett. B447, 199 (1999).

22. A. Deltuva, A.C. Fonseca, and P.U. Sauer, Phys. Rev. C 71, 054005 (2005).

23. A. Kievsky, S. Rosati, and M. Viviani, Nucl. Phys. A577, 511 (1994).

24. A. Kievsky, L.E. Marcucci, S. Rosati, and M. Viviani, Few-Body Syst. 22, 1 (1997).

25. M. Viviani, A. Kievsky, and S. Rosati, Phys. Rev. C 71, 024006 (2005).

26. M. Viviani et al. Few-Body Syst. 39, 159 (2006). 
27. N. Barnea, W. Leidemann, and G. Orlandini, Phys. Rev. C 61, 054001 (2000).

28. P. Navrátil and E. Caurier, Phys. Rev. C 69, 014311 (2004).

29. H. Kameyama, M. Kamimura, and Y. Fukushima, Phys. Rev. C 40, 974 (1989).

30. Y. Suzuki and K. Varga, Stochastic Variational Approach to Quantum Mechanical Few-Body Problems (Springer-Verlag, Berlin, 1998).

31. H. Kamada et al., Phys. Rev. C 64, 044001 (2001).

32. M. Fabre de la Ripelle, Ann. Phys. 147, 281 (1983).

33. A. Nogga et al., Phys. Rev. C 67, 034004 (2003).

34. A. Nogga, private communications.

35. A. Deltuva, R. Machleidt, and P.U. Sauer, Phys. Rev. C 68, 024005 (2003).

36. A. Deltuva, private communications.

37. R. Lazauskas and J. Carbonell, Phys. Rev. C 70, 044002 (2004).

38. D. Gazit et al., Phys. Rev. Lett. 96, 112301 (2006).

39. R. B. Wiringa et al., Phys. Rev. C 62, 014001 (2000).

40. P. Navrátil, private communications.

41. R. Machleidt, private communications.

42. W. Dilg, L. Koester, and W. Nistler, Phys. Lett. 36B, 208 (1971).

43. A.C. Phillips, Nucl. Phys. A107, 209 (1968)

44. J.L. Friar et al., Phys. Rev. C 30, 1121 (1984)

45. H.Rauch et al., Phys. Lett. 165B, 39 (1985).

46. G.M. Hale et al., Phys. Rev. C 42, 438 (1990).

47. R.B. Wiringa, R.A. Smith, and T.L. Ainsworth, Phys. Rev. C 29, 1207 (1984).

48. R.B. Wiringa (unpublished).

49. M. Viviani, S. Rosati, and A. Kievsky, Phys. Rev. Lett. 81, 1580 (1998).

50. K.A. Aniol et al., Phys. Rev. Lett. 82, 1096 (1999); Phys. Lett. B 509, 211 (2001); Phys. Rev. C 69, 065501 (2004); Phys. Lett. B 635, 275 (2006).

51. D.S. Amstrong et al., Phys. Rev. Lett. 95, 092001 (2005).

52. F.E. Maas et al., Phys. Rev. Lett. 93, 022002 (2004); Phys. Rev. Lett. 94, 152001 (2005).

53. D.T. Spayde et al., Phys. Lett. B 583, 79 (2004); T.M. Ito et al., Phys. Rev. Lett. 92, 102003 (2004).

54. K.A. Aniol et al., Phys. Rev. Lett. 96, 022003 (2006).

55. A. Acha et al., Phys. Rev. Lett. 98, 032301 (2007).

56. M.J. Musolf et al., Phys. Rep. 239, 1 (1994).

57. M.J. Musolf, R. Schiavilla, and T.W. Donnelly, Phys. Rev. C 50, 2173 (1994).

58. B. Kubis and R. Lewis, Phys. Rev. C 74, 015204 (2006).

59. M. A. Belushkin, H. W. Hammer and U.-G. Meißner, hep-ph/0608337; M. A. Belushkin, private communication.

60. S. Ramavataran, E. Hadjimichael, and T.W. Donnelly, Phys. Rev. C 50, 1175 (1994).

61. R.F. Frosch et al., Phys. Rev. 160, 874 (1968); R.G. Arnold et al., Phys. Rev. Lett. 40, 1429 (1978).

62. D.B. Leinweber et al., Phys. Rev. Lett. 97, 022001 (2006).

63. G. Miller, B.M.K. Nefkens, and I. Slaus, Phys. Rept. 194, 1 (1990). 